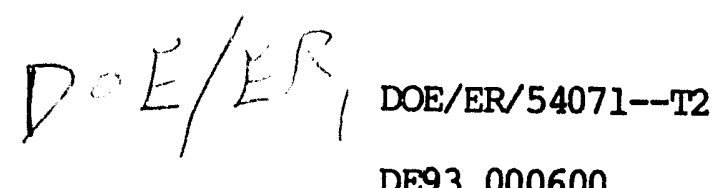

FY91 TECHNICAL PROGRESS REPORT

DOE PROJECT TITLE: SALIENT ISSUES OF EDGE PHYSICS PERTAINING TO LOSS OF CONFINEMENT:

A RESISTIVE MHD ANALYSIS

DOE GRANT \#:

P I :

ORGANIZATION:
DE-FG03-90ER54071

DR. DAVID R. THAYER

SAIC, SCIENCE APPLICATIONS INTERNATIONAL CORP.

\title{
TECHNICAL PROGRESS:
}

The progress that has been made during this fiscal year is significant in the area of tokamak edge plasma transport. We have made important contributions on the extension of the drift-rippling mode model $\left(\tilde{\phi}, \tilde{n}, \tilde{T}, \tilde{v}_{\|}\right)$ of edge turbulent transport. In particular, the research areas on which we have concentrated include the following topics: 1) The theoretical investigation of the radiatively enhanced transport due to the effects of impurity driven radiation instabilities has been expanded to include a situation with multiple impurities (such as carbon, $\mathrm{C}^{4+}$, and oxygen, $\mathrm{O}^{6+}$ ); 2) In order to validate the use of the impurity radiation input from the tokamak bolometer experiments in our theoretical edge turbulent transport calculations, the analysis that is utilized to transform impurity brightness data to radiated power profiles has been checked for state population and Abel inversion correctness; 3) The drift-rippling model of edge turbulent transport has been extended to include ionization particle sources in addition to the impurity driven thermal instability drive; and 4) The detailed limiter and realistic edge geometric effects on the edge turbulent transport has been included in the drift-rippling model. In addition to the work that we have conducted in the above listed areas, we are currently involved in extending these analyses to incorporate a twopoint theory of edge turbuient transport which will allow for direct calculation of the critical correlations (which include $\langle\tilde{n} \tilde{\phi}\rangle,\langle\tilde{n} \tilde{T}\rangle$, and $\langle\tilde{T} \tilde{\phi}\rangle$ ) that are needed for accurately comparing the experimentally determined transport coefficients with theory.

The first area of research that we have conducted in order to 
enhance the usefulness of the drift-rippling edge transport theory was to include multiple impurities in the radiation drive. The key to the impurity radiation drive is that it promotes instability in the temperature equation through two effects: the thermal instability and the condensation instability. In order to include the effects of multiple impurities which always exist in a realistic tokamak situation, the primitive drift-rippling theory has been enhanced by explicitly incorporating the impurity density equations along with a sum over the impurity radiation rates. In this case, the general radiation drive takes the form: $\partial \tilde{T} / \partial t=\ldots+\gamma_{T} \tilde{T}+\gamma_{n} \tilde{T}$, where the thermal instability, $\gamma_{T}=-(2 / 3) \sum_{z} n_{z 0} d I_{z}\left(T_{0}\right) / d T$, and the condensation instability, $\gamma_{n}=(2 / 3) \sum_{z} n_{z 0} I_{z}\left(T_{0}\right) / T$, include impurity summations. The critical feature of the radiation drive, related to the inclusion of the multiple impurity effects, is that this portion of the thermal instability can be additive or subtractive, due to the presence of the impurity radiation gradient with respect to temperature (or radial position). In particular, if the impurity radiation profile peaks farther out (radially from the main plasma) than the localization of the edge turbulent mode under consideration, then this "fickle" part of the radiation drive will be positive (which is not an uncommon effect of the carbon impurity on the dominant edge modes); however. if the impurity radiation profile peaks farther in (radially toward the main plasma) than the localization of the edge turbulent mode under consideration, then this "fickle" part of the radiation drive will be negative (which is not an uncommon effect of the oxygen impurity on the dominant edge modes). It should be noted that the condensation part of radiation drive is always additive; however, as previously noted, this instability mechanism is left relatively innocuous as a result of the lack of forced parallel pressure balance around the mode rational surface. Typically, the overall radiatively driven instability continues to persist in the multiple impurity scenario (although it may be enhanced or reduced depending on the details of the impurities present and the temperature profile). As an example of this multiple impurity effect, we have calculated the fluctuation level at saturation for the multiple impurity scenario with the carbon impurity radiation peaking at $10 \mathrm{eV}$, the oxygen impurity radiation peaking at $25 \mathrm{eV}$, and the drift rippling mode of interest peaking at a radial location which corresponds to a temperature of $15 \mathrm{eV}$. After performing a lengthy calculation, with the use of typical tokamak parameters and a shifted coronal radiation model $\left(R_{0}=1 \mathrm{~m}, B_{0}=2 \mathrm{~T}, T_{0}=10 \mathrm{eV}, n_{0}=5 \times 10^{18} \mathrm{~m}^{-3}, n_{z 0} / n_{0}=.02, V_{\text {Lexp }}=1 \mathrm{~V}, L_{s}=1.5 \mathrm{~m}\right.$, $L_{T}=.07 \mathrm{~m}, \eta_{n} \approx 1, \mathrm{~m} / \mathrm{n}=8 / 3$, and $r_{s} \approx .25 \mathrm{~m}$ ), the potential fluctuation level at saturation can be shown to be reduced to a level of $e \tilde{\phi} / T_{0} \approx 61$, which should 
be contrasted to a fluctuation level of $e \tilde{\phi} / T_{0} \approx .83$, if one considers solely the carbon impurity.

The second area of research that we have conducted in order to enhance the usefulness of the drift-rippling edge transport theory was to validate the use of the impurity radiation input from the tokamak bolometer experiments in our theoretical edge turbulent transport calculations. In particular, in order to determine the correctness of the tiansformation of the impurity brightness data to the radiated power profiles (which are used in the theoretical model), we have focused on the analysis of the upper state population and the Abel inversion of the emissivity. The radiation measurements produce values for the chord averaged brightness, $B$; however, in order to determine local levels of the emissivity, $\varepsilon$, an Abel inversion of the integral equation which describes the line of sight radiance has to be obtained so that the local radiated power, $P_{r a d}$, can be related to the impurity density and the line radiation intensity, $I_{k}$, where $P_{\text {rad }}(Z)=\sum n(Z) I_{k}$ and $n(Z)=f \varepsilon(Z)$. It should be noted that the analysis which has been performed is dependent on the assumption that the plasma is optically thin, allowing the radiation to freely escape so that it does not affect the level population. Furthermore, in order to account for intermediate densities, it was necessary to employ a collisional radiative model which is used to determine the population density, $N(k)$, that is mandatory for the calculation of the spectral radiation intensity. The integral equation for the brightness, $B(x)=2 \int d r r\left(r^{2}-x^{2}\right)^{-1 / 2} \bar{\varepsilon}(r)$, is inverted using standard techniques for the Abel integral equation. In particular, in addition to the inverse formula which contains a derivative of the experimentally determined brightness, $\bar{\varepsilon}(r)=-(1 / \pi) \int d x\left(x^{2}-r^{2}\right)^{-1 / 2} d B(x) / d r$, an additional derivative-free inversion formula has been utilized to alleviate the amplification of the errors which are inherent in the data. The population density is determined by the solution of the collisional radiative model, $d N(k) / d t=-N(k) \Gamma+\sum N\left(k^{\prime}\right) \Gamma+N^{\prime}(k) R$, which include decay and recombination probabilities. The upshot of this lengthy analysis is that the alteration in the experimentally determined impurity radiation profile which is used for the drift-rippling mode model of edge transport is within a $20 \%$ error bound of the previous profile that has been derived from the experiments. Furthermore, the radiation profile that is generated for use in the theoretical analysis produces results that are within $30 \%$ error of the results that can be obtained from shifted coronal radiation models. For example, the potential fluctuation at saturation is $e \tilde{\phi} / T_{0} \approx .65$, when this 
sophisticated radiation model has been employed; however, when the simple shifted coronal model is used, the result is $e \tilde{\phi} / T_{0} \approx 83$. Consequently, it is our recommendation that shifted coronal models (which have been previously employed) are sufficiently accurate for theoretical purposes since they produce accurate qualitative and semi-accurate quantitative results.

The third area of research that we have conducted in order to enhance the usefulness of the drift-rippling edge transport theory was to include ionization particle sources in addition to the impurity driven thermal instability drive. The sources that have been added to the driftrippling theory of edge turbulent transport have been used to explore various limiting cases of the theoretical framework in order to elucidate the essential features of the sources that have been considered. For the case of the impurity radiative drive, as we have described previously, due to the lack of parallel pressure balance around the mode rational surface, the radiation drive becomes essentially the thermal instability drive. In the limit that the temperature evolutions are tracked solely, the resultant drift-rippling dynamics reduces to essentially a rippling evolution ( $\tilde{T}$, dynamics), $\quad d \tilde{T} / d t-\chi \nabla_{\|}^{2} \tilde{T}=-\tilde{v}_{r} d T_{0} / d r+\gamma_{R} \tilde{T}$. Here, the radiation drive is simply the thermal instability, $\quad \gamma_{R} \rightarrow \gamma_{T}=-(2 / 3) n_{z 0} d I_{z}\left(T_{0}\right) / d T$, which represents the dominant growth of the mode for radial locations that correspond to the maximum of the impurity radiation gradient. In the limit that the potential and the temperature dynamics are tracked $(\tilde{\phi}-\tilde{T}$, dynamics), the resultant drift-rippling evolution is essentially that of the radiative drift wave. This reduction of the theoretical model is useful for stellarators (where $J_{100} \rightarrow 0$ and $\tilde{J}_{\| 1} \neq 0$ are allowed), and the result is consistent with the radiation dominant limit of the rippling saturated state, where $e \tilde{\phi} / T \propto \gamma_{R}^{3 / 2} /\left(\chi_{T} k_{11}^{2}\right)^{1 / 2}$. For the case of the density ionization source, an appropriate theoretical reduction of the analysis is to track the parallel velocity and the density fluctuations which result in an evolution of the ionization drift wave $\left(\tilde{\nu}_{\|}-\tilde{n}\right.$, dynamics). In this limit, the growth rate of the modes is dominantly the rate of the ionization, $\gamma \rightarrow \gamma_{l}$, and the particle flux is found to be inward, where the saturation level is proportional to the square of the growth rate, $\Gamma \sim-\gamma_{1}^{2} / \bar{k}^{2}$.

The fourth area of research that we have conducted in order to enhance the usefulness of the drift-rippling edge transport theory was to include the detailed limiter and realistic edge geometric effects on the edge turbulent transport. In order to appropriately cast the drift-rippling theory into an analysis which can be used to handle the realistic geometric 
constraints that exist in a limiter confined plasma, it is useful to transform to coordinates that are "along the field line". In this case, the Eikonal representation for the potential fluctuation takes on the form: $\tilde{\phi}(r, \theta, \varphi)=\sum_{m, n} \exp (\gamma t) \exp [i(n \varphi-m \theta)] \int d \xi \exp \{-i[n-m / q(r)] \xi\} \tilde{\phi}(\xi)$. The feature that is interesting and useful about using this transformation is that perpendicular derivatives become simply proportional to the along field line coordinate $\left[\nabla_{\perp}{ }^{2} \rightarrow-k_{\theta}{ }^{2}\left(R_{0} / L_{s}\right)^{2} \xi^{2}\right]$ and the parallel derivatives (which originally contained a mixture of $y$ and $z$ components) now are with respect to a single coordinate $\left(\nabla_{\|} \rightarrow R_{0} \partial / \partial \xi\right)$ wilich allows the incorporation of along field line boundary conditions using a trivial prescription. The set of nonlinear drift-rippling equations have been transformed by using this scheme so that the nonlinear saturated state could be analyzed for particular choices of detailed geometry. For the case of a poloidal limiter, the connection length along the field line is drastically altered which also influences the growth rate and the saturation level. In particular, if we consider a simplifying model which includes derivatives only up to second order, then the second order eigenmode equation can easily be solved, $\left[\partial^{2} / \partial \xi^{2}-i A \partial / \partial \xi-B \xi^{2}\right] \tilde{\phi}(\xi)=0, \quad$ where $\quad A=\eta_{0} R_{0} J_{z 0} k_{\theta} /\left(B_{0} L_{\eta} \gamma\right), \quad$ and $B=\eta_{0} \rho_{m} R_{0}{ }^{4} k_{\theta}{ }^{2} \gamma /\left(B_{z}^{2} L_{s}^{2}\right)$. In this case, the potential fluctuation growth rate can be determined by using a Kummers (confluent hypergeometric) function. The result of this analysis is that the growth rate is reduced by approximately an order of magnitude (stabilizing, $\gamma \rightarrow \gamma_{0} / 10$ ) and the nonlinear saturated state is reduced by approximately a factor of four $\left(e \tilde{\phi} / T_{0} \rightarrow .2\right)$. It should be noted that the basic mechanism for the reduction in the drift-rippling edge turbulent transport is that the geometric effect imposed on the boundary condition, due to limiter constraint, forces the modes to expand in width, so that the parallel thermal conduction dissipation is enhanced, and the modes are stabilized at lower levels than have been calculated previously.

Finally, the current area of drift-rippling edge turbulent transport theory on which we are focusing is the development of a two-point theory that will allow for direct calculation of the critical correlations (which include $\langle\tilde{n} \tilde{\phi}\rangle,\langle\tilde{n} \tilde{T}\rangle$, and $\langle\tilde{T} \tilde{\phi}\rangle$ ) that are needed for accurately comparing the experimentally determined transport coefficients with theory. Recently, there has been a great deal of experimental evidence which suggests that the geometric effects (such as the limiters) on the edge modes are substantial. In particular, the coherence and phase angle (as well as resultant correlation) of the field variables (such as the density and potential fluctuations) are not symmetric in the $r-\theta$ coordinates and are 
also critically effected by the limiter position. We have begun a theoretical exploration of this phenomena using the drift-rippling model to calculate the correlations directly from the theory in order to make further contact between theory and experiment. We are currently involved in solving (for certain limiting cases) the spectrally decomposed equations that describe the correlations which are needed for transport interpretation $\left(\left\langle\tilde{v}_{\left.r \bar{k}^{\prime}, \omega^{\prime}, \tilde{n}_{\bar{k}^{\prime \prime}, \omega^{*}}\right\rangle}\right\rangle\right.$ and $\left.\left\langle\tilde{v}_{r \bar{k}^{\prime}, \omega^{\prime}} \tilde{T}_{\bar{k}^{\prime \prime}, \omega^{\prime \prime}}\right\rangle\right)$. The form of the equation system is:

$\left(L_{T 1}+N L_{T 1}\right)\left\langle\tilde{v}_{r} \tilde{T}\right\rangle=L_{T 2}\left\langle\tilde{v}_{r} \tilde{n}\right\rangle+L_{T 3}\left\langle\tilde{v}_{r} \tilde{v}_{\| 1}\right\rangle+L_{T 4} \bar{v}_{r}^{2}$.

$\left(L_{n 1}+N L_{n 1}\right)\left\langle\tilde{v}_{r} \tilde{n}\right\rangle=L_{n 2}\left\langle\tilde{v}_{r} \tilde{v}_{\| 1}\right\rangle+L_{n 3} \bar{v}_{r}^{2}$,

$\left(L_{v 1}+N L_{v 1}\right)\left\langle\tilde{v}_{r} \tilde{v}_{11}\right\rangle=L_{v 2}\left\langle\tilde{v}_{r} \tilde{n}\right\rangle+L_{v 3}\left\langle\tilde{v}_{r} \tilde{T}\right\rangle$.

Here, $L_{\bar{k}^{\prime}, \omega^{\prime} ; \bar{k}^{\prime \prime}, \omega^{*}}$ are linear operators and $N L_{\vec{k}^{\prime}, \omega^{\prime} ; \bar{k}^{\prime \prime}, \omega^{\prime \prime}}$ are nonlinear operators. We will propose to conduct follow-on work to this analysis of the driftrippling model that will allow us to contribute towards a completion of this theoretical study of the critical field correlations which we have just recently begun to explore. Furthermore, we will also propose to incorporate the analysis of the detailed geometry effects on the theoretical correlation results. Once this suggested work has been completed, we will be able to compare (in detail) the edge turbulent transport theory with the experimental results which are just beginning to emerge.

This most recent tokamak edge transport work, that has been sponsored by DOE, has been presented and/or published in the following:

"Tokamak Edge Transport Issues," with P. H. Diamond, G. R. Tynan, et. al., Bull Am. Phys. Soc. 36, 2412 (1991).

"Influence of Material Boundaries on Tokamak Edge Transport," with P. H. Diamond, G. R. Tynan, et. al., to be published in Phys. Fluids B (1992).

"Influence of Impurities on Edge Turbulence and Transport in TEXT," with C. Hidalgo, C. P. Ritz, et al., Nuc. Fus. 31, 1661 (1991).

"Edge Turbulent Transport Critical Comparisons," with P. H. Diamond, G. R. Tynan, et. al., Sherwood Theory Meeting, Seattle, WA, ID28 (1991).

"Recent Edge Turbulent Transport Theory/Experiment Comparison Results," Transport Task Force Meeting, Austin, TX, (1991).

"Comparative Studies of Edge Turbulence in the TEXT Tokamak and the ATF Stellarator," with Ch. P. Ritz, T. L. Rhodes, et al., in Plasma Physics and 
Controlled Nuclear Fusion Research 1990, Proceedings of the 13th International Conference, Washington, DC (International Atomic Energy Agency, Vienna, 1991) 2, 589 (1991).

"Thermally Driven Turbulence and Transport in a Sheared System," with P. H. Diamond, Phys. Rev. Lett. 65, 2784 (1990).

"Tokamak Edge Transport Theory," with P. H. Diamond, A. S. Ware, et. al., Bull Am. Phys. Soc. 35, 1981 (1990).

\section{DISCLAIMER}

This report was prepared as an account of work sponsored by an agency of the United States Government. Neither the United States Government nor any agency thereof, nor any of their employees, makes any warranty, express or implied, or assumes any legal liability or responsibility for the accuracy, completeness, or usefulness of any information, apparatus, product, or process disclosed, or represents that its use would not infringe privately owned rights. Reference herein to any specific commercial product, process, or service by trade name, trademark, manufacturer, or otherwise does not necessarily constitute or imply its endorsement, recommendation, or favoring by the United States Government or any agency thereof. The views and opinions of authors expressed herein do not necessarily state or reflect those of the United States Government or any agency thereof. 

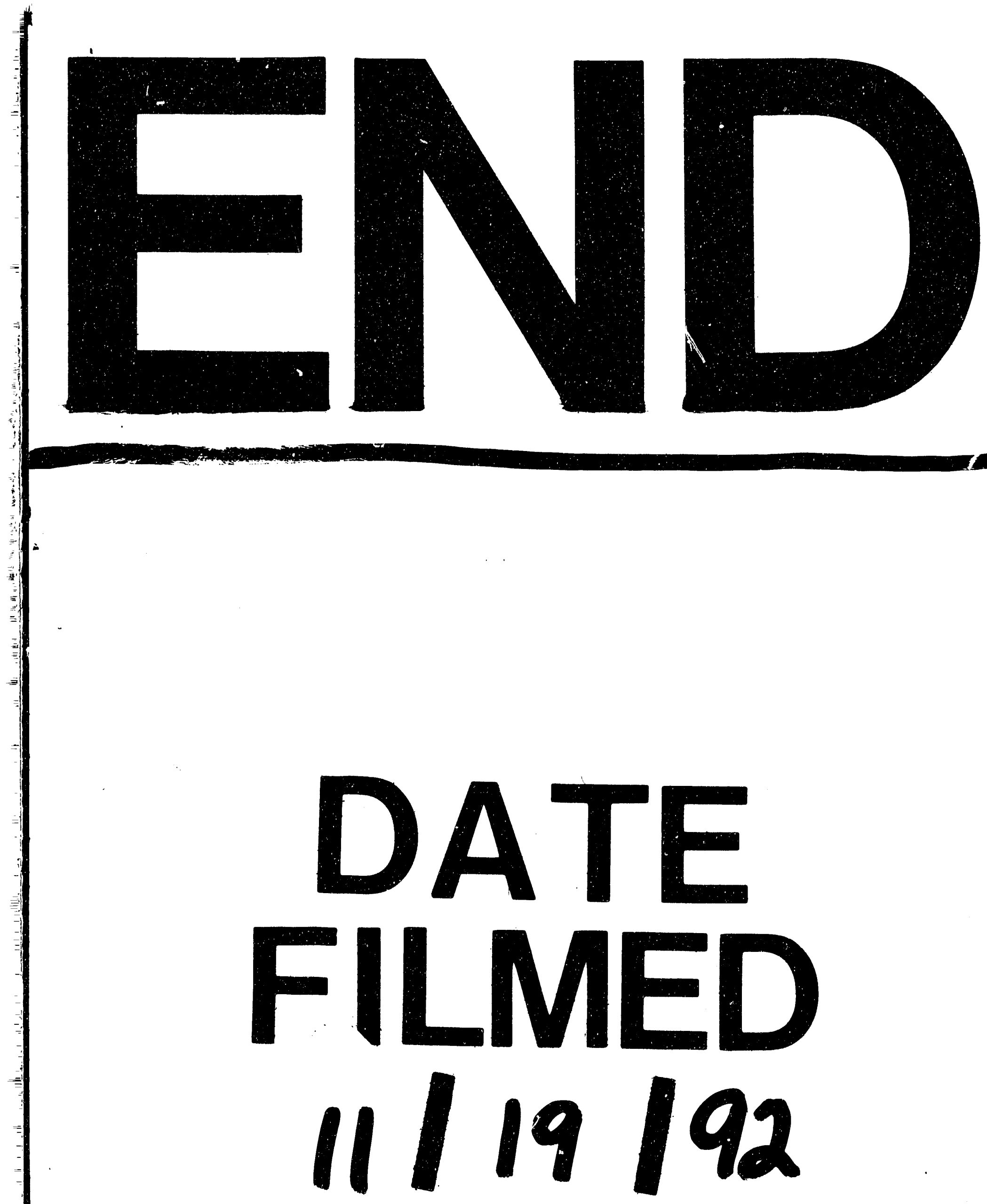
